\title{
Care of adults with neurofibromatosis type 1: a clinical practice resource of the American College of Medical Genetics and Genomics (ACMG)
}

\author{
Douglas R. Stewart, MD ${ }^{1}$, Bruce R. Korf, MD, Ph.D², Katherine L. Nathanson, MD ${ }^{3,4}$, \\ David A. Stevenson, $\mathrm{MD}^{5}$ and Kaleb Yohay, $\mathrm{MD}^{6}$
}

\begin{abstract}
Disclaimer: This practice resource is designed primarily as an educational resource for medical geneticists and other clinicians to help them provide quality medical services. Adherence to this practice resource is completely voluntary and does not necessarily assure a successful medical outcome. This practice resource should not be considered inclusive of all proper procedures and tests or exclusive of other procedures and tests that are reasonably directed to obtaining the same results. In determining the propriety of any

specific procedure or test, the clinician should apply his or her own professional judgment to the specific clinical circumstances presented by the individual patient or specimen.

Clinicians are encouraged to document the reasons for the use of a particular procedure or test, whether or not it is in conformance with this practice resource. Clinicians also are advised to take notice of the date this practice resource was adopted, and to consider other medical and scientific information that becomes available after that date. It also would be prudent to consider whether intellectual property interests may restrict the performance of certain tests and other procedures.
\end{abstract}

Purpose: Neurofibromatosis type 1 (NF1) is an autosomal dominant disorder that is caused by a heterozygous loss-offunction variant in the tumor suppressor gene NF1; it affects $\sim 1 / 1,900-1 / 3,500$ people worldwide. The disorder is associated with an 8-15-year reduction in average life expectancy in both men and women, primarily due to malignant neoplasms and cardiovascular causes.

Methods: A work group of experts sought to determine the prevalence, morbidity and mortality, and available treatments of common and emerging NF1-related clinical problems in adults. Work-group members identified peer-reviewed publications from PubMed. Publications derived from populations and multiinstitution cohorts were prioritized. Recommendations for management arose by consensus from this literature and the collective expertise of the authors.
Results: Malignant peripheral nerve sheath tumor (MPNST), breast cancer, cutaneous neurofibromas, and significant psychiatric and neurologic diagnoses are common problems in patients with NF1.

Conclusion: Patient education and sensitization to worrisome signs and symptoms such as progressive severe pain (MPNST), changes in tumor volume (MPNST), new, unexplained neurologic symptoms (MPNST, brain tumors), and diaphoresis/palpitations (pheochromocytoma) are important. Although many issues in adults with NF1 can be managed by an internist or family physician, we strongly encourage evaluation by, and care coordination with, a specialized NF1 clinic.

Genet Med advance online publication 26 April 2018

Key Words: breast cancer; cutaneous neurofibromas; malignant peripheral nerve sheath tumor; neurofibromatosis type 1; osteopenia/osteoporosis
Neurofibromatosis type 1 (NF1) is an autosomal dominant tumor-predisposition disorder that arises from loss-offunction variants in the tumor-suppressor gene $N F 1^{1,2}$. Features of the disorder typically appear in early childhood. It is fully penetrant by adulthood, although the spectrum of severity can vary considerably, even within families. It affects $\sim 1 / 1,900-1 / 3,500^{3}$ people worldwide. The disorder is associated with an 8-15-year reduction in average life expectancy in both men and women, due primarily to malignant neoplasms and cardiovascular causes. ${ }^{3,4}$ Most adults with NF1 are clinically diagnosed in childhood, according to NIH consensus criteria ${ }^{5}$ (Supplemental Table S1 online). The criteria are both highly specific and sensitive in adults with NF $1 .{ }^{6}$ In most cases, the diagnosis can be easily made based on a history, physical exam, and pedigree review and no additional imaging or NF1 genetic testing is needed. These issues and recommendations for the health supervision of children with NF1 are available elsewhere. ${ }^{7}$ In childhood, NF1

\footnotetext{
${ }^{1}$ Clinical Genetics Branch, Division of Cancer Epidemiology and Genetics, National Cancer Institute, NIH, Rockville, Maryland, USA; ${ }^{2}$ Department of Genetics, University of Alabama, Birmingham, Alabama, USA; ${ }^{3}$ Division of Translational Medicine and Human Genetics, Department of Medicine, Perelman School of Medicine at the University of Pennsylvania, Philadelphia, Pennsylvania, USA; ${ }^{4}$ Abramson Cancer Center, Perelman School of Medicine at the University of Pennsylvania, Philadelphia, Pennsylvania, USA; ${ }^{5}$ Department of Pediatrics, Division of Medical Genetics, Stanford University School of Medicine, Palo Alto, California, USA; ${ }^{6}$ Department of Neurology, New York University School of Medicine, New York, New York, USA. Correspondence: Douglas R. Stewart (drstewart@mail.nih.gov)

The Board of Directors of the American College of Medical Genetics and Genomics approved this clinical practice resource on 19 December 2017.

Submitted 22 January 2018; accepted 22 January 2018; advance online publication 26 April 2018. doi:10.1038/gim.2018.28
} 
genetic testing can quickly establish a diagnosis and relieve anxiety, but that is less likely an issue for adults. A limited number of NF1 genotype-phenotype correlations are known $^{8-11}$ and can be clinically useful in some cases. In general, however, the spectrum of severity of the NF1 phenotype, even within a family, can be striking.

There are many reasons why adults may present for care. These include:

1) assistance with a specific problem in a person with known NF1;

2) diagnostic evaluation of a parent with a child newly diagnosed with NF1;

3) guidance during pregnancy;

4) diagnostic evaluation because of clinical signs (e.g., caféau-lait macules);

5) diagnostic evaluation after detection of an NF1 variant of uncertain significance from a hereditary cancer predisposition gene panel.

\section{METHODS}

At the request of the American College of Medical Genetics and Genomics (ACMG) Professional Practice and Guidelines (PP\&G) committee, a work group of experts was convened in July 2015 to develop a Clinical Practice Resource for the clinical care of adults with NF1. Work-group members were selected based on published expertise in content areas relevant to care of an adult with NF1, including bone biology (DAS), genetics (BRK, KLN, DAS, DRS), malignancy (DRS, KLN, BRK, KY), breast cancer (KLN), neuropathy and chronic pain (BRK, DRS, KY), and vasculopathy (DRS, KY). A review of potential or actual conflicts of interest for each member was conducted as per ACMG policy. The group developed a list of common and emerging NF1-related clinical problems. For each problem, we sought to determine its prevalence, most common causes of morbidity and mortality, and available treatments. After outlining the key clinical areas to review, each expert assembled relevant peer-reviewed literature based on knowledge of the existing literature supplemented with additional searches of PubMed to identify any new publications (as of December 2016). We prioritized publications that studied populations, large clinic cohorts (preferably from multiple institutions), and large case series. We acknowledge that not all pertinent literature may have been found. Our recommendations for clinical management are derived by consensus from this literature and the collective expertise of the authors. Working and final drafts were reviewed and approved by members of the PP\&G committee and the ACMG Board of Directors. Guidance for the care of children with $\mathrm{NF}^{7}$ and advice for the transition from pediatric to adult care are also available. ${ }^{12}$

\section{MALIGNANCY}

\section{Malignant peripheral nerve sheath tumor}

In NF1, malignant peripheral nerve sheath tumor (MPNST), a type of soft-tissue sarcoma, frequently arises from a preexisting plexiform neurofibroma (PN), a benign, congenital lesion affecting approximately $50 \%$ of NF1 patients. ${ }^{13}$ MPNST is clinically aggressive and tends to metastasize early. A population-based NF1 patient series $(n=1,404 ; 19,076$ person-years' observation) linked to incident cancers in the Finnish Cancer Registry confirmed the dramatically increased risk of MPNST (standardized incidence ratio [SIR] = 2,056). ${ }^{14}$ The risk of NF1-associated MPNST to ages 30, 50, and 85 years was $8.5 \%, 12.3 \%$, and $15.8 \%$ respectively, as previously reported. ${ }^{15}$ These data also showed that high-grade MPNSTs were usually fatal, contributing significantly to NF1 mortality (standardized mortality ratio $>2,000$ ). No gender differences in survival were found. The Finnish data also showed no difference in MPNST survival in those with or without NF1. These results differ from a meta-analysis of $>1,800$ patients from 48 studies over five decades, which showed increased mortality for NF1 patients with MPNST compared with sporadic cases of MPNST, although outcomes have improved in the past decade ${ }^{16}$.

There are no pathognomonic molecular or immunohistochemical studies for MPNST and the histologic features are nonspecific. Low-grade MPNST accounts for 5\% of NF1associated MPNST and is associated with a 100\% ten-year survival, ${ }^{17}$ in contrast with high-grade tumors ( 20\% 5-year survival $\left.{ }^{14,15}\right)$. Surgery remains the cornerstone of treatment for high-grade MPNST, with the aim of achieving clear margins. This approach has demonstrated benefit even for large, non-extremity (e.g., abdominal) MPNST. ${ }^{18}$ We did not identify any randomized studies evaluating the benefit of adjuvant chemotherapy or radiation in MPNST, but a metaanalysis and recent studies suggest that there may be a role for them in some MPNST patients with non-metastatic disease. ${ }^{19}$ In advanced and metastatic MPNST, the prognosis is very poor; response rates to doxorubicin plus ifosfamide are $\sim 21 \%$ in a multi-institutional retrospective study. ${ }^{20}$

In two studies ( $n=148 ; n=130$ ) of NF1-associated MPNST, the median age at diagnosis was 33-34 years, about ten years younger than sporadic MPNST. ${ }^{17,21}$ Clinical suspicion (pain, rapid growth, neurologic symptoms, deep, truncal location of $\mathrm{PN}$ ), and awareness of known risk factors (germline microdeletion of the NF1 locus, ${ }^{22}$ previous radiation $^{23-25}$ ) remain paramount for early detection of MPNST, which is facilitated by targeted MRI imaging. The optimal use, timing, and utility of 18F-fluorodeoxyglucose positron emission tomography $\left({ }^{18} \mathrm{~F}-\mathrm{FDG} \mathrm{PET}\right), \mathrm{PET} / \mathrm{CT}$, and MRI (whole-body and focused studies) to screen for MPNST are not known. In the largest study of its kind to date (116 lesions, 59 with histologic confirmation) from 105 NF1 patients, Ferner and colleagues used ${ }^{18}$ F-FDG-PET and PET/ CT to diagnose NF1-associated MPNST with a sensitivity of 0.89 and a specificity of $0.95 .^{26}$ Review of 12 studies of 353 NF1 patients revealed that the maximum standardized uptake value $\left(\mathrm{SUV}_{\text {max }}\right)$ of 3.5 is a commonly accepted threshold for biopsy of a lesion, ${ }^{27}$ but this finding needs validation in large, prospective, randomized studies with histologic confirmation. The difference in $\mathrm{SUV}_{\max }$ in benign and malignant tumors is a function of imaging time after injection, and both early 
(90 minutes) and late (four hours) imaging can be used clinically. ${ }^{28}$

\section{SUMMARY}

(1) MPNST often arises from a PN. High-grade MPNSTs are usually fatal, frequently from metastasis.

(2) Clinical suspicion (progressive, persistent pain, and rapid growth) is key to diagnosis of MPNST. Early detection is essential for improved long-term survival.

(3) Diagnosis and grading of MPNST can be a challenge.

(4) Surgery is the primary treatment for high-grade MPNST and can be curative. The role of adjuvant chemotherapy and radiation needs further definition.

(5) The optimal use, timing, and utility of screening with whole-body MRI are not known.

\section{RECOMMENDATIONS}

- Consider referral to specialized high-volume centers for MPNST diagnosis and management.

- Imaging of an asymptomatic PN is a matter for clinical judgment. If a lesion involves only subcutaneous tissue with no deeper component, imaging may not be necessary.

- If a deeper component of a PN is suspected, imaging can be useful to assess the full extent of the lesion, to serve as a baseline to judge future growth, and to identify whether there is a nodular component that may be associated with an increased risk of malignant change. ${ }^{29}$

- MRI is preferred over CT scanning to reduce ionizing radiation exposure.

- Educate patients about MPNST signs and symptoms at initial and follow-up visits.

\section{Breast cancer}

Women with NF1 have an increased risk of breast cancer, which may present at an earlier age than in the general population. The Finnish population-based study of 1,404 persons with NF1 diagnosed clinically (19,076 person-years) from 1987 to 2012 demonstrated a significant excess of breast cancer in all women (SIR $=3.04 ; \mathrm{p}<0.001)$, especially in women under 40 years of age $(\operatorname{SIR}=11.1 ; \mathrm{p}<0.001)$, with the youngest cases diagnosed around age 30 . The investigators also reported significantly poorer five-year survival of patients with NF1-related breast cancer versus controls $(67.9 \%$ vs. $87.8 \% ; \mathrm{p}=0.004)$, as well as excess mortality (SMR = 5.20). ${ }^{14}$ Breast cancer death (proportionate mortality ratio) was 3.5-fold increased in women with NF1 (diagnosed clinically) over population controls in a study of 1,186 people with NF1 in northwest England. ${ }^{4}$ A study of hospitalization and mortality statistics in England ( 53 million people) from 1999 to 2011 identified 58 cases of breast cancer in 3,672 women with NF1. The investigators calculated relative and absolute risk per year in women with NF1; the highest rates were in women aged 30-39 years $(R R=6.5)$, decreasing to general population risk by age of around 70 years. $^{30}$ Histopathology review of 43 tumors from clinic- and population-based studies with available tissue showed a predominance of invasive ductal carcinoma, which is the most common subtype in the general population. ${ }^{31-35} \mathrm{We}$ did not find comprehensive studies investigating estrogen and progesterone receptor status or the frequency of concomitant pathogenic germline BRCA1/2 variants in people with NF1. Similarly, we could not find definitive studies on the risk of male breast cancer in NF1, although individual cases have been reported. ${ }^{36}$ Differences in chemotherapy efficacy, if any, in NF1-associated breast cancers warrants investigation. Although two studies describe women with NF1 and contralateral breast cancer, ${ }^{33,35}$ the risk and rate in the NF1 population are unknown.

Patients with a clinical diagnosis of NF1 are at moderately increased breast cancer risk, similar in magnitude to pathogenic variants in genes such as ATM, CHEK2, and $N B N .{ }^{37}$ Rare case reports document the concomitant carriage of pathogenic NF1 and breast cancer (BRCA1) alleles in the same person. ${ }^{38,39}$ One proposed strategy to clinically manage moderate-penetrance disorders that confer breast cancer risks similar to NF1 advocates initiating surveillance at the age when a woman's five-year risk approaches that at which screening is routinely initiated for women in the general population ( $\sim 1 \%$ in the USA). ${ }^{40}$ The National Comprehensive Cancer Network guidelines recommend an annual mammogram, starting at age 30 years, and consideration of contrast-enhanced breast MRI between ages 30 and 50 years for patients with NF1. These recommendations are based primarily on expert opinion rather than solid evidence, and thus warrant caution in their application. ${ }^{40}$ Research investigating the utility of mammography and breast MRI in women with NF1 is critically needed. Consideration should be given to the possibility of false positives arising on breast imaging from breast neurofibromas, which in turn leads to an increased number of breast biopsies. Multiple case reports have described delays in breast-cancer diagnosis due to cutaneous and breast neurofibromas that impair palpation of a concerning mass and the interpretation of mammography. ${ }^{41}$ The difficulty of imaging interpretation may contribute to the higher mortality observed in NF1-associated breast cancer. ${ }^{4,14}$

The threshold at which risk-reducing mastectomy should be considered in an at-risk but unaffected woman with NF1 is unknown. Such procedures are rarely performed on women with atypical ductal hyperplasia or lobular carcinoma in situ, conditions for which the average annual breast cancer risk is $\sim 1 \%$, a rate that is higher than that observed in carriers of moderate-penetrance breast cancer genes. ${ }^{40}$

\section{SUMMARY}

(1) Women with NF1 are at an increased risk for breast cancer, especially at $<40$ years; the earliest cases appear at $\sim 30$ years. The relative risk decreases with 
age, so that women with NF1 are at general population risk at $\sim 70$ years.

(2) Women with NF1-related breast cancer have a significantly poorer five-year survival and excess mortality.

(3) The predominant histology is invasive ductal carcinoma, the most common subtype in the general population.

(4) Contralateral breast-cancer risk, male breast-cancer rates, differences in chemotherapy efficacy, estrogen and progesterone receptor status, and specificity/ sensitivity of mammography and breast MRI merit additional study in NF1-associated breast cancer.

\section{RECOMMENDATIONS}

- National Comprehensive Cancer Network guidelines recommend an annual mammogram starting at age 30 years, and consideration of contrast-enhanced breast MRI between ages 30 and 50 years for women with a clinical diagnosis of NF1.

- Individualized shared decision-making on the timing and frequency of screening warrants consideration, given the absence of data on the performance characteristics of mammography or breast MRI in women with NF1.

- The decision to perform risk-reducing mastectomy should be guided by family history (e.g., the larger the number of first- and second-degree relatives with breast cancer, particularly early onset or bilateral breast cancer, the greater the breast-cancer risk to the patient).

- Consider the possibility of the coexistence of other breastcancer risk alleles (e.g., BRCA1/2) with NF1.

\section{Pheochromocytoma}

The incidence of pheochromocytoma in NF1 is estimated at $0.1-5.7 \% ; 22 \%$ are asymptomatic. ${ }^{42}$ The incidence in autopsy series is higher $(3.3-13.0 \%){ }^{42}$ A study of 25 patients from a pheochromocytoma registry found a median age of presentation of 43 years (range 14-61 years) with exclusively adrenal disease (no paragangliomas); $20 \%$ were multifocal and $12 \%$ were malignant, a rate similar to that in sporadic disease. ${ }^{43}$ Individuals with NF1 may harbor mixed pheochromocytoma and ganglioneuroma tumors. ${ }^{44}$ A second study of 48 NF1 patients evaluated at a hypertension clinic found that $15 \%$ harbored a pheochromocytoma; of these patients, 57\% were symptomatic at diagnosis. ${ }^{45}$

We did not find any randomized studies on the efficacy of biochemical or imaging screening to detect pheochromocytoma in asymptomatic patients with NF1. Measurement of plasma free metanephrine levels as a single test is more sensitive and specific than other studies in an NF1 patient clinically suspected to harbor a pheochromocytoma. ${ }^{46}$ Although localization of the pheochromocytoma is generally done by CT or MRI, functional imaging is helpful to detect multifocal disease, by either meta-iodobenzylguanidine or 3,4dihydroxy-6-18F-fluoro-L-phenylalanine positron emission tomography (FDOPA-PET). The latter was superior for detection of pheochromocytomas in patients with a hereditary pheochromocytoma syndrome in a small (25 patients; 64 lesions) non-randomized study. ${ }^{47}$ If laboratory testing is suggestive of pheochromocytoma with negative abdominal and pelvic imaging, meta-iodobenzylguanidine may be considered. In patients with pheochromocytomas, long-term follow-up includes annual surveillance with plasma metanephrines. ${ }^{48}$

\section{SUMMARY}

The median age of pheochromocytoma presentation in one study was 43 years (range 14-61 years) with exclusively adrenal disease (no paragangliomas); $20 \%$ were multifocal and $12 \%$ were malignant.

\section{RECOMMENDATIONS}

- Pheochromocytoma should be considered in hypertensive NF1 patients who are over 30 years of age, pregnant, and/ or have paroxysmal hypertension, hypertension-associated headache, palpitations, or sweating.

- Biochemical or imaging screening in asymptomatic patients with NF1 for pheochromocytoma is not recommended.

- Measurement of plasma free metanephrines as a single test is more sensitive and specific than other studies in an NF1 patient clinically suspected to harbor a pheochromocytoma. A follow-up 24-hour urine collection for catecholamines and metanephrines should be done if plasma testing is equivocal (less than fourfold elevation).

\section{Other malignancies}

NF1 causes an increased risk for several other tumor types. In the Finnish epidemiological study of 1,404 persons with NF1, other non-NF1-specific cancers for which a statistically significant excess has been found include those of the brain and central nervous system, "other endocrine glands," "illdefined or unknown," gastrointestinal stromal tumors (GIST), malignant fibrous histiocytoma, and rhabdomyosarcoma. ${ }^{14}$ An excess of deaths was reported for thyroid and "ill-defined or unknown" malignancies in this cohort. The excess of brain tumors included higher grade, non-optic pathway gliomas. An epidemiologic analysis of adult glioma $(n=489)$ and adolescent and adult NF1 $(n=2,108)$ found a 20-100-fold increased risk (vs. US rates) of developing malignant glioma, including glioblastoma multiforme. ${ }^{49}$ The increased risk for tumors of the endocrine organs in the Finnish study may arise from periampullary somatostatinomas, a type of carcinoid tumor that is estimated to affect $1 \%$ of people with NF1 and has been observed to cluster with GISTs and pheochromocytomas. ${ }^{50}$ The association in NF1 with GIST (adult, typically small intestine and multifocal) ${ }^{50}$ and rhabdomyosarcoma (pediatric, typically urogenital) ${ }^{50}$ has been well established. The association with thyroid cancer and malignant fibrous histiocytoma was unexpected. The 
number of people affected with each of these cancers was small ( $\leq 5$ in a study of 1,404 Finns with NF1 over 25 years); this observation needs replication in other large populationbased studies. In the Finnish study, there was no excess of leukemia or lymphoma, including juvenile myelomonocytic leukemia, despite an established genetic association with NF1 in children. ${ }^{51}$

\section{SUMMARY}

Malignancies with epidemiologic evidence of association with NF1 include malignant gliomas, GISTs, rhabdomyosarcoma, thyroid, and malignant fibrous histiocytoma. Tumors with genetic evidence of association include pheochromocytoma, somatostatinomas, and juvenile myelomonocytic leukemia.

\section{RECOMMENDATIONS}

We are not aware of evidence supporting special screening for rarer NF1-associated malignancies in asymptomatic patients.

\section{Hypertension and vasculopathy}

Hypertension is commonly observed in persons with NF1, and its incidence increases with age. ${ }^{52}$ The most common causes of hypertension in NF1 are essential hypertension, pheochromocytoma (above), and, in young adults, renal artery stenosis. ${ }^{52}$ Renal artery abnormalities may be seen in up to $1 \%$ of individuals with NF1. ${ }^{53}$ For patients with a glomerular filtration rate (GFR) $>30 \mathrm{~mL} / \mathrm{min}$, contrastenhanced magnetic resonance angiography (MRA) is highly sensitive and specific. ${ }^{54,55}$ The incidence of stroke (predominantly hemorrhagic) may be increased in NF1 compared with the general population. ${ }^{56}$ Pregnant women with NF1 may have additional risk of hypertension and cerebrovascular complications compared with pregnant women without NF1. ${ }^{57}$

NF1-associated vasculopathy affects small, medium, and large vessels. The arterial vasculature is most often affected, although venous and pulmonary artery involvement also may occur. ${ }^{58} \mathrm{NF1-associated} \mathrm{vasculopathy} \mathrm{is} \mathrm{frequently} \mathrm{asympto-}$ matic, and thus the incidence, spectrum, and natural history of these lesions are poorly characterized. Types of associated vascular abnormalities include arterial stenosis, moyamoya arteriopathy, aneurysms, arteriovenous malformations, cardiac valve abnormalities, and vessel compression and/or invasion by neurofibromatosis-related tumors. ${ }^{59}$ The overall incidence of vasculopathy in NF1 is estimated to be between $0.4 \%$ and $6.4 \% ;^{52,60,61}$ the incidence specifically of cerebrovascular vasculopathy is estimated to be $2 \%$ to $5 \% .{ }^{62}$ In a retrospective review of reported cases of NF1 vasculopathy, renal artery stenosis was most commonly reported (41\%), followed by cerebrovascular vasculopathy (19\%) and abnormalities of the abdominal aorta (12\%). ${ }^{59}$ A vasculopathy is presumed to underlie NF1-associated pulmonary hypertension, a rare but potentially fatal complication. ${ }^{58} \mathrm{We}$ did not find prospective studies of the incidence and natural history of NF1-related vasculopathy and we could not find data on the role of routine vascular screening in adults with NF1.
Plexiform neurofibromas are highly vascular and can spontaneously bleed, sometimes seriously enough to be lifethreatening. The hemorrhage may clinically present as a rapidly growing tumor. Patients who undergo surgery to remove a plexiform neurofibroma may have a significant intra-operative transfusion requirement, given the challenges of maintaining adequate hemostasis in these tumors.

\section{SUMMARY}

(1) The most common causes of elevated blood pressure in NF1 are essential hypertension, pheochromocytoma, and, in young adults, renal artery stenosis. Pregnant women with NF1 may have additional risk of hypertension and cerebrovascular complications.

(2) The incidence of stroke (predominantly hemorrhagic) may be increased in NF1.

(3) NF1-associated vasculopathy affects small, medium, and large vessels (primarily arteries); venous and pulmonary artery involvement also may occur.

(4) NF1-associated vasculopathy is frequently asymptomatic; the incidence, spectrum, and natural history of these lesions are poorly characterized.

(5) The overall incidence of vasculopathy in NF1 is estimated to be between $0.4 \%$ and $6.4 \%$; the incidence specifically of cerebrovascular vasculopathy is estimated to be $2 \%$ to $5 \%$.

\section{RECOMMENDATIONS}

- For hypertensive NF1 patients who are under 30 years of age, pregnant and/or have abdominal bruits, causes of renovascular hypertension should be first evaluated (see Supplemental Figure S1).

- MRA is the preferred imaging modality for evaluation of renovascular hypertension. However, for patients with impaired renal function (GFR $<30 \mathrm{~mL} / \mathrm{min}$ ), spiral CT and CT angiography may be used. If imaging is negative, consider formal renal angiography. Concomitant screening for pheochromocytoma with plasma free metanephrines is also recommended.

- Treatment for NF1-associated hypertension should be tailored to the specific etiology.

- Selective imaging should be performed for patients in whom there is clinical suspicion of a vascular lesion.

- For identified vascular abnormalities, decisions regarding timing and type of intervention should be the same as for patients without NF1.

\section{Bone health}

Musculoskeletal findings are frequent in NF1; the presence of a "distinctive osseous lesion" is one of the consensus diagnostic criteria. ${ }^{5}$ The most morbid skeletal manifestations are focal lesions such as long bone dysplasia/pseudarthrosis, dystrophic scoliosis, and sphenoid wing dysplasia, which 
typically present in childhood but can have lasting effects into adulthood.

Decreased bone mineral density and osteoporosis have been documented in multiple studies as having a high prevalence in NF1 adults. ${ }^{63-66}$ Several studies report an increased fracture rate in NF1 adults, ${ }^{67-69}$ with one study showing a 5.2-fold increase in relative risk for NF1 individuals $>41$ years of age compared with a control cohort of individuals who had undergone emergency appendectomy in Finland. ${ }^{69}$ It has been proposed that Vitamin D deficiency contributes to osteoporosis in NF1. ${ }^{65,67,68,70}$ The impact of vitamin D supplementation in NF1 on bone density and fractures is unclear. ${ }^{65,68}$ We were unable to find published evidence of a proven association of parathyroid disease with NF1.

Osteoporosis occurs at an earlier age in adults with $\mathrm{NF}^{66}$ and progresses over time. ${ }^{66,71}$ Screening using dual-energy $\mathrm{X}$-ray absorptiometry (DXA) is utilized in the general population based on various protocols depending on age, sex, hormonal status, and other risk factors. Earlier screening with DXA may be warranted in individuals with NF1, but data are lacking. There are guidelines in the general population for initiation of bisphosphonate therapy based on DXA values and clinical history using several fracture risk assessment tools, and NF1 should be entered as a risk factor when using such algorithms. ${ }^{72-74}$ One study of six people with $\mathrm{NF} 1$ and osteoporosis treated with alendronate observed an increase in bone mineral density in five of six individuals after 23 months of treatment. ${ }^{71}$ The increase did not reach statistical significance, however, and one subject sustained a new stress fracture. In vitro studies have shown that osteoclasts derived from individuals with NF1 display reduced sensitivity to bisphosphonates, raising question about the clinical effectiveness and dosing of these agents in NF1. ${ }^{75}$

Scoliosis in NF1 is typically categorized as nondystrophic and dystrophic. ${ }^{76}$ The dystrophic form typically presents in childhood, whereas the nondystrophic form can develop into adulthood. Significant scoliosis-associated spinal pathology includes dural ectasia, vertebral degenerative changes, and spinal compression. Curve progression is typically more rapid in those with dystrophic features. ${ }^{77} \mathrm{~A}$ study of 17 young adults with $\mathrm{NF}^{78}$ found that approximately $50 \%$ of those with scoliosis diagnosed prior to adulthood had progression of curvature, with significant back pain. In addition, $18 \%$ of the young adults had developed a new mild scoliosis.

\section{SUMMARY}

(1) The most morbid skeletal manifestations (long bone dysplasia/pseudarthrosis, dystrophic scoliosis, and sphenoid wing dysplasia) typically present in childhood but can have lasting effects into adulthood.

(2) Decreased bone-mineral density and osteoporosis have been documented in multiple studies as having a high prevalence in NF1 adults. Osteoporosis occurs at an earlier age in adults with NF1 and progresses over time.
(3) Scoliosis in NF1 is historically categorized as nondystrophic and dystrophic. Significant scoliosisassociated spinal pathology includes dural ectasia, vertebral degenerative changes, and spinal compression.

\section{RECOMMENDATIONS}

- Although lacking published evidence of efficacy, we recommend vitamin $\mathrm{D}$ supplementation in individuals with NF1 to reach serum 25-hydroxyvitamin D concentrations in the sufficient range.

- Osteoporosis occurs at an earlier age in adults with NF1 and progresses over time. Treatment is not different from what is recommended in the general population.

- Management of scoliosis in NF1 adults consists of monitoring for curve progression. All individuals with NF1 should have annual clinical evaluation of the back with Adam's forward bend test and be referred to orthopedics if there is concern about scoliosis.

\section{Neurofibroma burden and cosmesis}

Cutaneous neurofibromas (cNF) can occur on the surface or within the skin. cNFs are histologically similar to PN but do not appear to undergo malignant transformation. ${ }^{79}$ Although cNFs are not life-threatening, they can lead to significant morbidity. A population-based survey in Wales found cNFs in $>99 \%$ of adults with $\mathrm{NF} 1,{ }^{80}$ making this the most common tumor manifestation in adults. This study described an increase in the number of cNFs with increasing age and revealed that 10 of $30(33 \%)$ women underwent an increase in size and number of cNFs during pregnancy. Another study investigating patient-reported pregnancy outcomes in women with NF1 found 64 of $105(60 \%)$ developed new cNFs and 55 of 105 (52\%) reported cNF size increases. ${ }^{81}$ Further, a crosssectional study of 721 pediatric and adult patients with NF1 showed that the number of cNFs strongly increased with age. ${ }^{82}$ Collectively, these studies indicate that $\mathrm{cNF}$ often begin to appear in puberty, continue to increase in number with age, and display periods of rapid growth during puberty and pregnancy.

The growth and number of cNFs may impose a cosmetic burden, may be symptomatic (tenderness, bleeding, itching), and may be responsible for negative effects on quality of life. A quality of life survey completed by 128 adults with NF1 in France showed that visibility of lesions was associated with negative effects on emotions, physical symptoms, and functioning. ${ }^{83}$ Similar findings were reported in Italian and U.S. cohorts. ${ }^{84,85}$

Current treatment options for cNF include surgical excision, laser removal, or electrodesiccation. We are not aware of published evidence to guide choice amongst these options. Surgical excision typically targets a modest number of symptomatic $\mathrm{CNFs}^{86}$ and leaves a scar. Laser removal of cNFs has been used for several decades and encompasses photocoagulation and $\mathrm{CO}_{2}$ laser ablation; radiofrequency 
ablation is also used. ${ }^{87-92}$ Anesthesia is required to remove 50-100 superficial lesions per session. A retrospective study queried 106 people with NF1 about pain and treatment safety after $\mathrm{CO}_{2}$ laser treatment; ${ }^{93}>90 \%$ were satisfied with the treatment and reported minimal pain, good healing, and few complications (e.g., bleeding, scarring, infection). Electrodesiccation dehydrates and denatures the dermis. A retrospective study of 97 individuals with NF1 who had electrodesiccation of cutaneous and subcutaneous cNFs in one or more stages ${ }^{94}$ found that a mean of 450 lesions were removed per session. Overall, there was minimal scarring, minor discomfort, and high patient satisfaction at the postoperative visit. Similar findings were reported in another retrospective study of six individuals with NF1 undergoing electrodessication. ${ }^{95}$ Additional cNFs will appear over time and repeated treatments will be required. We are not aware of nonsurgical therapies for cNF. Progress in the identification of agents controlling the growth of $\mathrm{PN}^{96}$ suggests that similar agents for the control of cNF may be possible, especially given the histologic similarities between the two tumors.

In a small study of French adults with NF1, 70\% reported daily or almost-daily moderate-intensity pruritus, typically in the evenings. About half had pruritus localized to neurofibromas. ${ }^{97} \mathrm{~A}$ small minority $(<10 \%)$ regularly used an emollient or oral antihistamine, with limited effect. ${ }^{97}$

\section{SUMMARY}

(1) cNFs can occur on the surface or within the skin, and very rarely, if ever, undergo malignant transformation.

(2) cNFs often begin to appear in puberty, increase in number with age, and display periods of rapid growth during puberty and pregnancy.

(3) cNFs may impose a cosmetic burden, may be symptomatic (tenderness, bleeding, itching) and are associated with negative effects on emotions, physical symptoms, and functioning.

\section{RECOMMENDATIONS}

- Current treatment options for cNF include surgical excision, laser removal, or electrodesiccation. Physician and patient preference and local expertise typically guide choice of these options.

- Minimal scarring, minor discomfort, and high patient satisfaction have been reported with all three therapies. General anesthesia may be required for the removal of many lesions.

- Nonsurgical therapies (medication and devices) are under development but are not clinically available at this time.

- NF1-associated pruritus may be neuropathic in origin and thus may be responsive to localized and generalized therapies (including gabapentin and pregabalin), but NF1-specific data are lacking. ${ }^{98}$

\section{Neurocognitive and psychiatric problems DEPRESSION}

Studies have demonstrated an increased prevalence of depression and other psychiatric disorders, with at least one third of adult NF1 patients affected. One cross-sectional study found significant depressive symptoms in 55\% of 498 adults self-reported as having NF1. ${ }^{99}$

\section{ADULT ATTENTION DEFICIT AND HYPERACTIVITY DISORDER (ADHD)}

ADHD is well characterized in the pediatric NF1 population. We found limited data on the prevalence or characterization of ADHD in adults with NF1, though several studies have demonstrated impaired performance on attentional tests in adults with NF1. ${ }^{100-102}$ One study has shown lower quality of life and emotional stability in adults with NF1 and ADHD than in adults with NF1 alone. ${ }^{103}$

\section{COGNITIVE IMPAIRMENT}

Cognitive impairment is well-characterized in children with NF1 and persists into adulthood, ${ }^{100}$ affecting, in our experience, school performance and employability. Studies have demonstrated that IQ may be reduced in adults compared to the general population, to a similar extent to that of children with NF1. ${ }^{101,102}$ One study of 20 adults with NF1 compared to age-matched controls (spouses of subjects) demonstrated deficits in visual-spatial skills, memory, selective attention, and components of executive function. ${ }^{101}$ We found limited data on cognitive function in elderly adults with NF1, though one small study demonstrated cognitive impairments similar to those found in children and younger adults with NF1. ${ }^{104}$ Microdeletion of the NF1 gene is associated with more significant intellectual impairment. ${ }^{105}$

\section{RECOMMENDATIONS}

- Consider screening for depression in adults with NF1, with appropriate referral.

- There are limited data on the prevalence of ADHD in adults with NF1. ADHD can affect employability and schooling.

- More severe cognitive impairment merits thorough evaluation (e.g., microarray, fragile $\mathrm{X}$ testing, concomitant monogenic disorders) for other etiologies.

\section{Chronic neurological conditions, neuropathy, and pain}

A study of private health-insurance data in NF1 children and adults $(n=8,579)$ and age-and gender-matched non-NF1 adults and children $(n=85,790)$ found significantly higher odds of submission of claims for headache, headache with migraine, epilepsy, and sleep disturbance/disorder in NF1 adults. ${ }^{106}$ This association remained significant after excluding individuals with brain-tumor-related claims. The age at first health-care claim for headache and headache with migraine was significantly younger than the non-NF1 population, but there were no age differences observed for epilepsy. The study also 
found significantly higher odds of an adult NF1 healthinsurance claim for Parkinson's disease and multiple sclerosis; however, the number of observations was small and there is a need for replication. ${ }^{106}$ A clinic-based study of headache in 115 adults with NF1 found that migraine aura, symptom triggers, age of onset, and phenotype mirror similar features in the general population. ${ }^{107} \mathrm{~A}$ prospective questionnaire study of 114 adults with NF1 (but no controls) found a high frequency of sleep disturbance, impaired sleep, and excessive daytime sleepiness. ${ }^{108}$ We did not identify any large studies evaluating differences in treatment of NF1-associated migraine, seizures, or sleep disorders.

A distinct, rare (2-3\%), adult-onset, non-progressive, symmetrical polyneuropathy, originally termed "neurofibromatous neuropathy," has been described. ${ }^{109}$ We were unable to find data from large cohorts. Now referred to as "NF1 neuropathy," it features a typically indolent, nerve-lengthdependent sensorimotor polyneuropathy with predominantly sensory signs and modestly abnormal electrophysiological findings. It is painless in the majority of patients ${ }^{109}$ and may arise from nerve microlesions. ${ }^{110}$

Glomus tumors are small, benign, but painful tumors of the glomus body, a thermoregulatory shunt in the fingertips, and are part of the NF1 phenotype. ${ }^{111}$ These tumors should not be confused with paragangliomas, which have also been called glomus tumors in the older literature. The majority of people affected have at least two of three symptoms of the classic triad of localized tenderness, severe paroxysmal pain, and sensitivity to cold. ${ }^{112}$ Women are more commonly affected than men and multifocal tumors are common. ${ }^{12}$ Clinical suspicion is essential; MRI imaging may be useful. ${ }^{112}$ Although surgery can be curative, delay in diagnosis (years) is not uncommon and can lead to chronic pain from the complex regional pain syndrome. ${ }^{112}$

We were unable to find high-quality studies investigating pain in adults with NF1. In our experience, chronic pain in NF1 adults is common, and negatively affects quality of life. NF1related pain may be nociceptive or neuropathic and can arise from PN, scoliosis, or pseudarthrosis. Frequently, however, no anatomic correlate can be identified. New-onset, increasingly severe pain should be heeded as a possible symptom of an MPNST (see section on MPNST). It is important to remember that not all pain in individuals with NF1 is due to NF1, and common etiologies (e.g., lower back pain) remain common.

Young adults with NF1, a chronic neurologic condition, may inquire about their eligibility to serve in the armed forces. In the United States, Army Regulation 40-501 ("Standards of Medical Fitness," guidance used by all branches of the US military), notes that a history of neurofibromatosis is a disqualification for enlistment.

\section{SUMMARY}

(1) Headache, headache with migraine, epilepsy, and sleep disturbance/disorder are more common in NF1 adults, based on insurance-claim data.
(2) A tumor-independent NF1 neuropathy is recognized but rare and varied in symptomology and progression.

(3) Chronic pain is probably an underrecognized problem in NF1. Chronic pain from fingertip glomus tumors is rare but treatable.

\section{RECOMMENDATIONS}

- NF1-associated migraine, seizures, or sleep disorders should be treated as in the non-NF1 population.

- Medication, physical therapy, and surgery (for compressive tumors) may be beneficial to NF1 neuropathy.

- Adults with NF1 should be queried about chronic fingertip and toe pain in the assessment of possible glomus tumors.

- Screening and use of pain-interference scales may be useful, with referral to a pain clinic as needed, preferably those that employ both pharmacologic and nonpharmacologic approaches.

\section{Contraception, pregnancy, and assisted fertility}

A 1988 study of all individuals with "neurofibromatosis" in Gothenburg, Sweden found a reduction in fertility by $\sim 20 \%$ and ascribed the difference to social rather than biologic factors. ${ }^{113}$ There are limited data on the safety of oral or depot contraceptives in women with NF1. However, the prevention of unintended pregnancy is important, and thus the benefits likely outweigh any theoretical concerns. Similarly, if oral or depot contraceptives are required to treat a medical condition, the benefits are likely greater than any theoretical concerns. Expression of progesterone receptors has been found in 75\% of neurofibromas. ${ }^{114}$ One survey of 59 women with NF1 found that 53 (89\%) who received oral estrogen-progestogen or pure progestogen preparations reported no associated neurofibroma growth. Two women (3\%) in the study who received depot contraceptives containing a high dose of synthetic progesterone reported significant tumor growth. ${ }^{115}$ A retrospective, population-based cohort study in the USA of 19 million pregnancy-related admissions from 1988 to 2009 (1553 associated with NF1) found that NF1 was associated with a statistically significant excess of maternal morbidity (gestational hypertension, preeclampsia, intrauterine growth restriction, cerebrovascular disease, preterm labor, and cesarean delivery), but not maternal mortality. ${ }^{57}$ We did not find literature describing significant adverse events or outcomes in women with NF1 undergoing epidural anesthesia. In one study of 105 women (247 pregnancies), $55-60 \%$ of women reported an increase in growth of new or existing cNFs during pregnancy and 30\% observed postpartum regression of neurofibroma size. ${ }^{81}$ A study of 156 cycles of preimplantation genetic diagnosis (PGD) in couples with NF1 showed a lower proportion resulting in live birth (versus nonNF1 couples), but the rate was comparable with that seen in couples with other autosomal dominant or X-linked disorders. ${ }^{116}$ Prenatal diagnosis is possible through fetal 
Table 1 Assessment of adults with NF1

\begin{tabular}{l} 
In addition to recommended age-and gender-specific screening and vaccinations, an annual general medical evaluation of the adult with NF1 should \\
consider questions about: \\
Medical history \\
Signs and symptoms of 1) malignant peripheral nerve sheath tumor, 2) pheochromocytoma, 3) neuropathy, 4) depression, 5) chronic pain and pruritus, 6) \\
fingertip pain \\
Bothersome/symptomatic cutaneous neurofibromas \\
Family planning/contraception (and referral for genetic counseling if needed) \\
Physical exam \\
Blood pressure \\
Clinical evaluation for scoliosis with Adam's forward bend test with referral if needed \\
Laboratory investigation \\
Consider in context of clinical presentation and age: serum vitamin D concentrations and supplementation \\
Imaging \\
Mammogram (women): start annually at age 30 years \\
MRI breast with contrast (women): consider between ages 30 and 50 years ${ }^{\text {a }}$ \\
Consider baseline MRI of known or suspected nonsuperficial plexiform neurofibromas \\
Consider in context of clinical presentation and age: dual energy X-ray absorptiometry \\
\hline
\end{tabular}

a Based on expert recommendations made by the National Comprehensive Cancer Network. The evidence base for these guidelines in women with NF1 is minimal. These suggestions were developed by analogy, based on the current approach to breast cancer screening in other intermediate-risk breast cancer susceptibility syndromes. Caution is warranted in applying these guidelines to NF1 patients.

DNA sequencing (obtained via amniocentesis or chorionic villus sampling) of known disease-causing NF1 mutations. ${ }^{117}$ Prenatal ultrasound diagnosis of NF1 has been reported, but would be feasible only in the setting of an NF-related lesion that is visible by ultrasound, which accounts for only a small proportion of cases. ${ }^{118}$

\section{RECOMMENDATIONS}

- If pregnancy is not desired, contraception should be used.

- Referral to a high-risk obstetrician should be considered for pregnant women with NF1.

- Preanesthesia neuraxial imaging to evaluate for spinal or paraspinal neurofibromas is probably not needed. If there are concerns, spinal anesthesia may be considered.

- Educate adults with NF1 that, as an autosomal dominant disorder, the offspring recurrence risk is $50 \%$ for each pregnancy.

- PGD and prenatal diagnosis of NF1 are available. Individuals with de novo mutations, somatic mosaicism, and large genomic rearrangements are much less likely to be able to have PGD owing to technical limitations.

\section{Concluding recommendations}

In this Clinical Practice Resource, we have emphasized studies that investigated populations, multi-institution clinic cohorts, and large case series. We acknowledge that not all relevant publications may have been identified. Table 1 lists suggested items to review at initial and follow-up visits for an adult with NF1. In addition, general population age- and gender-specific health screening (e.g., for cholesterol, colonoscopy, for cervical cancer) and vaccination schedules should be followed, regardless of NF1 diagnosis. Supplemental Resources lists useful websites and organizations. Although it is important to always consider NF1-associated etiologies for a new sign or symptom, common explanations will remain common (e.g., most hypertension is essential and not pheochromocytomarelated, most back pain will not be due to an MPNST, most headaches will not be tumor-associated). Repeated patient education and sensitization to worrisome signs and symptoms such as progressive severe pain (MPNST), changes in tumor volume (MPNST), new, unexplained neurologic symptoms (MPNST, CNS tumors), and diaphoresis/palpitations (pheochromocytoma) are important. ${ }^{12}$ Although many issues in adults with NF1 can be managed by an internist or family physician, we strongly encourage evaluation by and care coordination with a specialized NF1 clinic. (The Children's Tumor Foundation (see Supplemental Resources) sponsors the NF Clinic Network, a nationwide network of NF clinics.) For some patients, a multidisciplinary NF clinic may serve best as a medical home, although all patients should have a primary care physician. In addition, through an NF clinic, patients may more easily learn of research studies aimed to develop novel therapies. To improve clinical care of adults with NF1, future research efforts need to address, among other issues, the sensitivity and specificity of breast MRI and mammography in NF1, the role of whole-body and/or targeted MRI as a screen for MPNST, the full spectrum of NF1-associated malignancy, the natural history and treatment of NF1-associated osteoporosis and osteopenia, treatment of chronic pain, NF1-associated Parkinson's disease and multiple sclerosis, and effective biomarkers and imaging strategies for tumor screening (e.g., pheochromocytoma, glioblastoma multiforme). 


\section{SUPPLEMENTARY MATERIAL}

Supplementary material is linked to the online version of the paper at http://www.nature.com/gim

\section{ACKNOWLEDGMENTS}

This work was supported by the Intramural Research Program of the Division of Cancer Epidemiology and Genetics of the National Cancer Institute, Bethesda, MD. K.N. acknowledges grants from the Abramson Cancer Center (P30 CA016520) and from the Breast Cancer Research Foundation. We thank Vincent Riccardi, MD, Xia Wang, MD (Moffitt Cancer Center), and Ashley Cannon, MS, PhD (UAB) for comments on the manuscript.

\section{DISCLOSURE}

D.A.S. has been a consultant for and received compensation from Lineagen, Alexion, and GLG. B.R.K. has been a consultant for Novartis and received a travel grant from AstraZeneca. The other authors declare no conflict of interest.

\section{REFERENCES}

1. Wallace MR, Marchuk DA, Andersen LB, et al. Type 1 neurofibromatosis gene: identification of a large transcript disrupted in three NF1 patients. Science 1990;249:181-186.

2. Viskochil D, Buchberg $A M, X u G$, et al. Deletions and a translocation interrupt a cloned gene at the neurofibromatosis type 1 locus. Cell 1990;62:187-192.

3. Uusitalo E, Leppavirta J, Koffert A, et al. Incidence and mortality of neurofibromatosis: a total population study in Finland. J Invest Dermatol. 2015;135:904-906.

4. Evans DG, O'Hara C, Wilding A, et al. Mortality in neurofibromatosis 1: in North West England: an assessment of actuarial survival in a region of the UK since 1989. Eur J Hum Genet. 2011;19:1187-1191.

5. National Institutes of Health Consensus Development Conference Statement: neurofibromatosis. Bethesda, Md., USA, 13-15 July, 1987. Neurofibromatosis 1988;1:172-178.

6. Friedman JM. Neurofibromatosis 1. In: Pagon RA, Adam, M.P., Ardinger, H.H. et al., ed. GeneReviews. Seattle: University of Washington, Seattle; 2014.

7. Hersh JH, American Academy of Pediatrics Committee on Genetics. Health supervision for children with neurofibromatosis. Pediatrics 2008;121:633-642.

8. Mautner VF, Kluwe L, Friedrich RE, et al. Clinical characterisation of 29 neurofibromatosis type-1 patients with molecularly ascertained $1.4 \mathrm{Mb}$ type-1 NF1 deletions. J Med Genet. 2010;47:623-630.

9. Upadhyaya M, Huson SM, Davies $M$, et al. An absence of cutaneous neurofibromas associated with a 3-bp inframe deletion in exon 17 of the NF1 gene (c.2970-2972 delAAT): evidence of a clinically significant NF1 genotype-phenotype correlation. Am J Hum Genet. 2007;80:140-151.

10. Pinna $V$, Lanari $V$, Daniele $P$, et al. p.Arg1809Cys substitution in neurofibromin is associated with a distinctive NF1 phenotype without neurofibromas. Eur J Hum Genet. 2015;23:1068-1071.

11. Burkitt Wright EM, Sach E, Sharif S, et al. Can the diagnosis of NF1 be excluded clinically? A lack of pigmentary findings in families with spinal neurofibromatosis demonstrates a limitation of clinical diagnosis. J Med Genet. 2013;50:606-613.

12. Van Lierde A, Menni F, Bedeschi MF, et al. Healthcare transition in patients with rare genetic disorders with and without developmental disability: neurofibromatosis 1 and Williams-Beuren syndrome. Am J Med Genet A. 2013;161A:1666-1674.

13. Mautner VF, Asuagbor FA, Dombi E, et al. Assessment of benign tumor burden by whole-body MRI in patients with neurofibromatosis 1. Neuro Oncol. 2008;10:593-598.

14. Uusitalo E, Rantanen M, Kallionpaa RA, et al. Distinctive cancer associations in patients with neurofibromatosis type 1. J Clin Oncol. 2016.

15. Evans DG, Baser ME, McGaughran J, Sharif S, Howard E \& Moran A. Malignant peripheral nerve sheath tumours in neurofibromatosis 1. J Med Genet. 2002;39:311-314.
16. Kolberg M, Holand M, Agesen TH, et al. Survival meta-analyses for 1800 malignant peripheral nerve sheath tumor patients with and without neurofibromatosis type 1. Neuro Oncol. 2013;15:135-147.

17. Watson KL, Al Sannaa GA, Kivlin CM, et al. Patterns of recurrence and survival in sporadic, neurofibromatosis Type 1-associated, and radiationassociated malignant peripheral nerve sheath tumors. I Neurosurg 2017;126:319-329.

18. Dunn GP, Spiliopoulos K, Plotkin SR, et al. Role of resection of malignant peripheral nerve sheath tumors in patients with neurofibromatosis type 1. J Neurosurg 2013;118:142-148.

19. Farid $M$, Demicco EG, Garcia R, et al. Malignant peripheral nerve sheath tumors. Oncologist 2014;19:193-201.

20. Kroep JR, Ouali M, Gelderblom H, et al. First-line chemotherapy for malignant peripheral nerve sheath tumor (MPNST) versus other histological soft tissue sarcoma subtypes and as a prognostic factor for MPNST: an EORTC soft tissue and bone sarcoma group study. Ann Oncol 2011:22:207-214

21. Valentin T, Le Cesne A, Ray-Coquard I, et al. Management and prognosis of malignant peripheral nerve sheath tumors: The experience of the French Sarcoma Group (GSF-GETO). Eur J Cancer. 2016;56:77-84.

22. De Raedt $T$, Brems $H$, Wolkenstein $P$, et al. Elevated risk for MPNST in NF1 microdeletion patients. Am J Hum Genet. 2003;72:1288-1292.

23. Ducatman BS \& Scheithauer BW. Postirradiation neurofibrosarcoma. Cancer 1983;51:1028-1033.

24. Meadows AT, Baum E, Fossati-Bellani F, et al. Second malignant neoplasms in children: an update from the Late Effects Study Group. J Clin Oncol. 1985;3:532-538.

25. Ducatman BS, Scheithauer BW, Piepgras DG, Reiman HM \& Ilstrup DM. Malignant peripheral nerve sheath tumors. A clinicopathologic study of 120 cases. Cancer 1986;57:2006-2021.

26. Ferner RE, Golding JF, Smith M, et al. [18F]2-fluoro-2-deoxy-D-glucose positron emission tomography (FDG PET) as a diagnostic tool for neurofibromatosis 1 (NF1) associated malignant peripheral nerve sheath tumours (MPNSTs): a long-term clinical study. Ann Oncol 2008;19: 390-394.

27. Treglia G, Taralli S, Bertagna F, et al. Usefulness of whole-body fluorine18-fluorodeoxyglucose positron emission tomography in patients with neurofibromatosis type 1: a systematic review. Radiol Res Pract. 2012;2012:431029.

28. Warbey VS, Ferner RE, Dunn JT, Calonje E \& O'Doherty MJ. [18F]FDG PET/ $\mathrm{CT}$ in the diagnosis of malignant peripheral nerve sheath tumours in neurofibromatosis type-1. Eur J NuCl Med Mol Imaging. 2009;36: 751-757.

29. Meany H, Dombi E, Reynolds J, et al. 18-fluorodeoxyglucose-positron emission tomography (FDG-PET) evaluation of nodular lesions in patients with neurofibromatosis type 1 and plexiform neurofibromas (PN) or malignant peripheral nerve sheath tumors (MPNST). Pediatr Blood Cancer. 2013:60:59-64.

30. Seminog $\mathrm{OO} \&$ Goldacre MJ. Age-specific risk of breast cancer in women with neurofibromatosis type 1. Br J Cancer. 2015;112:1546-1548.

31. Zoller ME, Rembeck B, Oden A, Samuelsson M \& Angervall L. Malignant and benign tumors in patients with neurofibromatosis type 1 in a defined Swedish population. Cancer 1997;79:2125-2131.

32. Walker $L$, Thompson $D$, Easton $D$, et al. A prospective study of neurofibromatosis type 1 cancer incidence in the UK. Br J Cancer. 2006;95: 233-238.

33. Sharif S, Moran A, Huson SM, et al. Women with neurofibromatosis 1 are at a moderately increased risk of developing breast cancer and should be considered for early screening. J Med Genet. 2007;44:481-484.

34. Madanikia SA, Bergner A, Ye X \& Blakeley JO. Increased risk of breast cancer in women with NF1. Am J Med Genet A. 2012;158A: 3056-3060.

35. Wang X, Levin AM, Smolinski SE, Vigneau FD, Levin NK \& Tainsky MA. Breast cancer and other neoplasms in women with neurofibromatosis type 1: a retrospective review of cases in the Detroit metropolitan area. Am J Med Genet A. 2012;158A:3061-3064.

36. Lakshmaiah KC, Kumar AN, Purohit $S$, et al. Neurofibromatosis type I with breast cancer: not only for women! Hered Cancer Clin Pract. 2014;12:5

37. Easton DF, Pharoah PD, Antoniou AC, et al. Gene-panel sequencing and the prediction of breast-cancer risk. N Engl J Med. 2015;372:2243-2257.

38. Ceccaroni M, Genuardi M, Legge F, et al. BRCA1-related malignancies in a family presenting with von Recklinghausen's disease. Gynecol Oncol 2002:86:375-378. 
39. Campos B, Balmana J, Gardenyes J, et al. Germline mutations in NF1 and BRCA1 in a family with neurofibromatosis type 1 and early-onset breast cancer. Breast Cancer Res Treat. 2013;139:597-602.

40. Tung N, Domchek SM, Stadler Z, et al. Counselling framework for moderate-penetrance cancer-susceptibility mutations. Nat Rev Clin Oncol. 2016;13:581-588.

41. Da Silva AV, Rodrigues FR, Pureza M, Lopes VG \& Cunha KS. Breast cancer and neurofibromatosis type 1: a diagnostic challenge in patients with a high number of neurofibromas. BMC Cancer 2015;15:183.

42. Walther MM, Herring J, Enquist E, Keiser HR \& Linehan WM. Von Recklinghausen's disease and pheochromocytomas. J Urol 1999;162: 1582-1586.

43. Bausch B, Borozdin W \& Neumann HP, European-American pheochromocytoma study G. Clinical and genetic characteristics of patients with neurofibromatosis type 1 and pheochromocytoma. N Engl J Med. 2006;354:2729-2731.

44. Mezitis SG, Geller M, Bocchieri E, Del Pizzo J \& Merlin S. Association of pheochromocytoma and ganglioneuroma: unusual finding in neurofibromatosis type 1. Endocr Pract. 2007;13:647-651.

45. Zinnamosca L, Petramala L, Cotesta D, et al. Neurofibromatosis type 1 (NF1) and pheochromocytoma: prevalence, clinical and cardiovascular aspects. Arch Dermatol Res. 2011;303:317-325.

46. Lenders JW, Pacak K, Walther MM, et al. Biochemical diagnosis of pheochromocytoma: which test is best? JAMA 2002;287:1427-1434.

47. Fottner C, Helisch A, Anlauf $M$, et al. 6-18F-fluoro-L-dihydroxyphenylalanine positron emission tomography is superior to 1231 metaiodobenzyl-guanidine scintigraphy in the detection of extraadrenal and hereditary pheochromocytomas and paragangliomas: correlation with vesicular monoamine transporter expression. J Clin Endocrinol Metab. 2010;95:2800-2810.

48. Amar L, Servais A, Gimenez-Roqueplo AP, Zinzindohoue F, Chatellier G \& Plouin PF. Year of diagnosis, features at presentation, and risk of recurrence in patients with pheochromocytoma or secreting paraganglioma. J Clin Endocrinol Metab. 2005;90:2110-2116.

49. Gutmann DH, Rasmussen SA, Wolkenstein P, et al. Gliomas presenting after age 10 in individuals with neurofibromatosis type 1 (NF1). Neurology 2002;59:759-761.

50. Patil S \& Chamberlain RS. Neoplasms associated with germline and somatic NF1 gene mutations. Oncologist 2012;17:101-116.

51. Shannon KM, O'Connell P, Martin GA, et al. Loss of the normal NF1 allele from the bone marrow of children with type 1 neurofibromatosis and malignant myeloid disorders. N Engl J Med. 1994;330:597-601.

52. Friedman JM, Arbiser J, Epstein JA, et al. Cardiovascular disease in neurofibromatosis 1: report of the NF1 Cardiovascular Task Force. Genet Med 2002;4:105-111.

53. Finley JL \& Dabbs DJ. Renal vascular smooth muscle proliferation in neurofibromatosis. Hum Pathol 1988;19:107-110.

54. Postma CT, Joosten FB, Rosenbusch $G$ \& Thien $T$. Magnetic resonance angiography has a high reliability in the detection of renal artery stenosis. Am J Hypertens. 1997;10(9 Pt 1):957-963.

55. Schoenberg SO, Rieger J, Johannson LO, et al. Diagnosis of renal artery stenosis with magnetic resonance angiography: update 2003. Nephrol Dial Transplant. 2003;18:1252-1256.

56. Terry AR, Jordan JT, Schwamm L \& Plotkin SR. Increased risk of cerebrovascular disease among patients with neurofibromatosis type 1 : population-based approach. Stroke 2016;47:60-65.

57. Terry AR, Barker 2nd FG, , Leffert L, Bateman BT, Souter I \& Plotkin SR. Neurofibromatosis type 1 and pregnancy complications: a populationbased study. Am J Obstet Gynecol. 2013;209:46 e41-48.

58. Stewart DR, Cogan JD, Kramer MR, et al. Is pulmonary arterial hypertension in neurofibromatosis type 1 secondary to a plexogenic arteriopathy? Chest 2007;132:798-808.

59. Oderich GS, Sullivan TM, Bower TC, et al. Vascular abnormalities in patients with neurofibromatosis syndrome type I: clinical spectrum, management, and results. J Vasc Surg. 2007;46:475-484.

60. Hamilton SJ \& Friedman JM. Insights into the pathogenesis of neurofibromatosis 1 vasculopathy. Clin Genet. 2000;58:341-344.

61. Lin $A E$, Birch PH, Korf BR, et al. Cardiovascular malformations and other cardiovascular abnormalities in neurofibromatosis 1. Am J Med Genet. 2000;95:108-117.

62. Cairns AG \& North KN. Cerebrovascular dysplasia in neurofibromatosis type 1. J Neurol Neurosurg Psychiatry. 2008;79:1165-1170.

63. Kuorilehto T, Poyhonen M, Bloigu R, Heikkinen J, Vaananen K \& Peltonen J. Decreased bone mineral density and content in neurofibromatosis type 1: lowest local values are located in the load-carrying parts of the body. Osteoporos Int. 2005;16:928-936.

64. Lammert M, Kappler M, Mautner VF, et al. Decreased bone mineral density in patients with neurofibromatosis 1. Osteoporos Int. 2005;16: 1161-1166.

65. Seitz S, Schnabel C, Busse B, et al. High bone turnover and accumulation of osteoid in patients with neurofibromatosis 1. Osteoporos Int. 2010;21: 119-127.

66. Heerva E, Leinonen P, Kuorilehto T, et al. Neurofibromatosis 1-related osteopenia often progresses to osteoporosis in 12 years. Calcif Tissue Int. 2013;92:23-27

67. Tucker T, Schnabel C, Hartmann M, et al. Bone health and fracture rate in individuals with neurofibromatosis 1 (NF1). J Med Genet. 2009;46: 259-265.

68. Brunetti-Pierri N, Doty SB, Hicks J, et al. Generalized metabolic bone disease in neurofibromatosis type I. Mol Genet Metab. 2008;94:105-111.

69. Heerva $E$, Koffert A, Jokinen $E$, et al. A controlled register-based study of 460 neurofibromatosis 1 patients: increased fracture risk in children and adults over 41 years of age. J Bone Miner Res. 2012;27:2333-2337.

70. Lammert M, Friedman JM, Roth HJ, et al. Vitamin D deficiency associated with number of neurofibromas in neurofibromatosis 1. J Med Genet. 2006;43:810-813.

71. Heerva $E$, Huilaja L, Leinonen $P$, Peltonen $S$ \& Peltonen J. Follow-up of six patients with neurofibromatosis 1-related osteoporosis treated with alendronate for 23 months. Calcif Tissue Int. 2014;94:608-612.

72. Crandall CJ. Risk assessment tools for osteoporosis screening in postmenopausal women: a systematic review. Curr Osteoporos Rep. 2015;13:287-301.

73. Rotondi NK, Beaton DE, Elliot-Gibson V, et al. Comparison of CAROC and FRAX in fragility fracture patients: agreement, clinical utility, and implications for clinical practice. J Rheumatol. 2016;43:1593-1599.

74. Pecina JL, Romanovsky L, Merry SP, Kennel KA \& Thacher TD. Comparison of clinical risk tools for predicting osteoporosis in women ages 50-64. J Am Board Fam Med. 2016;29:233-239.

75. Heerva E, Peltonen S, Svedstrom E, Aro HT, Vaananen K \& Peltonen J. Osteoclasts derived from patients with neurofibromatosis 1 (NF1) display insensitivity to bisphosphonates in vitro. Bone 2012;50:798-803.

76. Tsirikos Al, Saifuddin A \& Noordeen MH. Spinal deformity in neurofibromatosis type-1: diagnosis and treatment. Eur Spine J. 2005;14: 427-439.

77. Durrani AA, Crawford AH, Chouhdry SN, Saifuddin A \& Morley TR. Modulation of spinal deformities in patients with neurofibromatosis type 1. Spine (Phila Pa 1976). 2000;25:69-75.

78. Oates EC, Payne JM, Foster SL, Clarke NF \& North KN. Young Australian adults with NF1 have poor access to health care, high complication rates, and limited disease knowledge. Am J Med Genet A. 2013;161A: 659-666.

79. Carroll SL \& Ratner N. How does the Schwann cell lineage form tumors in NF1? Glia 2008;56:1590-1605.

80. Huson SM, Harper PS \& Compston DA. Von Recklinghausen neurofibromatosis. A clinical and population study in south-east Wales. Brain 1988;111 (Pt 6):1355-1381.

81. Dugoff L \& Sujansky E. Neurofibromatosis type 1 and pregnancy. Am J Med Genet. 1996;66:7-10.

82. Duong TA, Bastuji-Garin S, Valeyrie-Allanore L, Sbidian E, Ferkal S \& Wolkenstein P. Evolving pattern with age of cutaneous signs in neurofibromatosis type 1: a cross-sectional study of 728 patients. Dermatology 2011;222:269-273.

83. Wolkenstein P, Zeller J, Revuz J, Ecosse E \& Leplege A. Quality-of-life impairment in neurofibromatosis type 1: a cross-sectional study of 128 cases. Arch Dermatol 2001;137:1421-1425.

84. Kodra Y, Giustini S, Divona L, et al. Health-related quality of life in patients with neurofibromatosis type 1. A survey of 129 Italian patients. Dermatology 2009;218:215-220.

85. Page PZ, Page GP, Ecosse E, Korf BR, Leplege A \& Wolkenstein P. Impact of neurofibromatosis 1 on quality of life: a cross-sectional study of 176 American cases. Am J Med Genet A. 2006;140:1893-1898.

86. Bromley GS, Sherman JE \& Goulian Jr. D, Neurofibromatosis-distribution of lesions and surgical treatment. Ann Plast Surg. 1982;8:272-276.

87. Becker Jr. DW, Use of the carbon dioxide laser in treating multiple cutaneous neurofibromas. Ann Plast Surg. 1991;26:582-586.

88. Elwakil TF, Samy NA \& Elbasiouny MS. Non-excision treatment of multiple cutaneous neurofibromas by laser photocoagulation. Lasers Med Sci. 2008;23:301-306. 
89. Kim HJ, Lee KG, Yi SM, Kim JH \& Kim IH. Successful treatment of multiple cutaneous neurofibromas using a combination of shave excision and laser photothermocoagulation with a 1,444-nm neodymium-doped yttrium aluminum garnet laser. Dermatol Surg 2012;38:960-963.

90. Kriechbaumer LK, Susani M, Kircher SG \& Happak W. Vaporization of cutaneous neurofibromas with an erbium: yttrium-aluminum-garnet laser: a comparative histologic evaluation. Plast Reconstr Surg. 2012;129: 602e-604e.

91. Moreno JC, Mathoret C, Lantieri L, Zeller J, Revuz J \& Wolkenstein P. Carbon dioxide laser for removal of multiple cutaneous neurofibromas. $\mathrm{Br}$ J Dermatol. 2001;144:1096-1098.

92. Roenigk RK \& Ratz JL. CO2 laser treatment of cutaneous neurofibromas. J Dermatol Surg Oncol. 1987;13:187-190.

93. Meni C, Sbidian E, Moreno JC, et al. Treatment of neurofibromas with a carbon dioxide laser: a retrospective cross-sectional study of 106 patients. Dermatology 2015;230:263-268.

94. Levine SM, Levine E, Taub PJ \& Weinberg H. Electrosurgical excision technique for the treatment of multiple cutaneous lesions in neurofibromatosis type I. J Plast Reconstr Aesthet Surg. 2008;61:958-962.

95. Lutterodt CG, Mohan A \& Kirkpatrick N. The use of electrodessication in the treatment of cutaneous neurofibromatosis: A retrospective patient satisfaction outcome assessment. J Plast Reconstr Aesthet Surg. 2016;69: 765-769.

96. Gutmann DH, Blakeley JO, Korf BR \& Packer RJ. Optimizing biologically targeted clinical trials for neurofibromatosis. Expert Opin Investig Drugs. 2013;22:443-462.

97. Brenaut E, Nizery-Guermeur C, Audebert-Bellanger S, et al. Clinical characteristics of pruritus in neurofibromatosis 1. Acta Derm Venereol. 2016;96:398-399

98. Misery L, Brenaut E, Le Garrec R, et al. Neuropathic pruritus. Nat Rev Neurol. 2014;10:408-416.

99. Cohen JS, Levy HP, Sloan J, Dariotis J \& Biesecker BB. Depression among adults with neurofibromatosis type 1: prevalence and impact on quality of life. Clin Genet 2015;88:425-430.

100. Pavol M, Hiscock M, Massman P, Moore lii B, Foorman B \& Meyers C. Neuropsychological function in adults with von Recklinghausen's neurofibromatosis. Dev Neuropsychol. 2006;29:509-526.

101. Descheemaeker MJ, Plasschaert E, Frijns JP \& Legius E. Neuropsychological profile in adults with neurofibromatosis type 1 compared to a control group. J Intellect Disabil Res. 2013;57:874-886.

102. Ferner RE, Hughes RA \& Weinman J. Intellectual impairment in neurofibromatosis 1. J Neurol Sci. 1996;138:125-133.

103. Mautner VF, Granstrom S \& Leark RA. Impact of ADHD in adults with neurofibromatosis type 1: associated psychological and social problems. J Atten Disord. 2015;19:35-43.
104. Costa Dde S, de Paula JJ, de Rezende NA, et al. Neuropsychological impairments in elderly neurofibromatosis type 1 patients. Eur J Med Genet. 2014;57:216-219.

105. Tonsgard JH, Yelavarthi KK, Cushner S, Short MP \& Lindgren V. Do NF1 gene deletions result in a characteristic phenotype? Am J Med Genet. 1997;73:80-86.

106. Madubata CC, Olsen MA, Stwalley DL, Gutmann DH \& Johnson KJ. Neurofibromatosis type 1 and chronic neurological conditions in the United States: an administrative claims analysis. Genet Med 2015;17: 36-42.

107. Afridi SK, Leschziner GD \& Ferner RE. Prevalence and clinical presentation of headache in a national neurofibromatosis 1 service and impact on quality of life. Am J Med Genet A. 2015;167A: 2282-2285.

108. Leschziner GD, Golding JF \& Ferner RE. Sleep disturbance as part of the neurofibromatosis type 1 phenotype in adults. Am J Med Genet $A$ 2013:161A:1319-1322.

109. Ferner RE, Hughes RA, Hall SM, Upadhyaya M \& Johnson MR. Neurofibromatous neuropathy in neurofibromatosis 1 (NF1). J Med Genet. 2004;41:837-841.

110. Schulz A, Grafe P, Hagel C, et al. Neuropathies in the setting of neurofibromatosis tumor syndromes: complexities and opportunities. Exp Neurol 2017.

111. Brems $H$, Park C, Maertens $O$, et al. Glomus tumors in neurofibromatosis type 1: genetic, functional, and clinical evidence of a novel association. Cancer Res 2009:69:7393-7401.

112. Stewart DR, Sloan JL, Yao L, et al. Diagnosis, management, and complications of glomus tumours of the digits in neurofibromatosis type 1. J Med Genet. 2010;47:525-532.

113. Samuelsson B \& Akesson HO. Relative fertility and mutation rate in neurofibromatosis. Hereditas 1988;108:169-171.

114. McLaughlin ME \& Jacks T. Progesterone receptor expression in neurofibromas. Cancer Res 2003;63:752-755.

115. Lammert M, Mautner VF \& Kluwe L. Do hormonal contraceptives stimulate growth of neurofibromas? A survey on 59 NF1 patients. BMC Cancer 2005:5:16.

116. Merker VL, Murphy TP, Hughes JB, et al. Outcomes of preimplantation genetic diagnosis in neurofibromatosis type 1. Fertil Steril 2015;103: 761-768 e761.

117. van Minkelen $R$, van Bever $Y$, Kromosoeto JN, et al. A clinical and genetic overview of 18 years neurofibromatosis type 1 molecular diagnostics in the Netherlands. Clin Genet 2014;85:318-327.

118. McEwing RL, Joelle R, Mohlo M, Bernard JP, Hillion $Y$ \& Ville $Y$. Prenata diagnosis of neurofibromatosis type 1: sonographic and MRI findings. Prenat Diagn 2006;26:1110-1114. 